\title{
Shisa3 brakes resistance to EGFR-TKls in lung adenocarcinoma by suppressing cancer stem cell properties
}

\author{
Jiahui $\mathrm{Si}^{\dagger}$, Yuanyuan $\mathrm{Ma}^{\dagger}$, Ji Wang Bi, Ying Xiong, Chao Lv, Shaolei Li, Nan Wu and Yue Yang ${ }^{*}$
}

\begin{abstract}
Background: Although EGFR tyrosine kinase inhibitors (EGFR-TKIs) are beneficial to lung adenocarcinoma patients with sensitive EGFR mutations, resistance to these inhibitors induces a cancer stem cell (CSC) phenotype. Here, we clarify the function and molecular mechanism of shisa3 as a suppressor that can reverse EGFR-TKI resistance and inhibit CSC properties.
\end{abstract}

Methods: The suppresser genes involved in EGFR-TKI resistance were identified and validated by transcriptome sequencing, quantitative real-time PCR (qRT-PCR) and immunohistochemistry. Biological function analyses, cell half maximal inhibitory concentration (IC50), self-renewal, and migration and invasion capacities, were detected by CCK8, sphere formation and Transwell assays. Tumorigenesis and therapeutic effects were investigated in nonobese diabetic/severe combined immunodeficiency (nod-scid) mice. The underlying mechanisms were explored by Western blot and immunoprecipitation analyses.

Results: We found that low expression of shisa3 was related to EGFR-TKI resistance in lung adenocarcinoma patients. Ectopic overexpression of shisa3 inhibited CSC properties and the cell cycle in the lung adenocarcinoma cells resistant to gefitinib/osimertinib. In contrast, suppression of shisa3 promoted CSC phenotypes and the cell cycle in the cells sensitive to EGFR-TKIs. For TKI-resistant PC9/ER tumors in nod-scid mice, overexpressed shisa3 had a significant inhibitory effect. In addition, we verified that shisa3 inhibited EGFR-TKI resistance by interacting with FGFR1/3 to regulate AKT/mTOR signaling. Furthermore, combinational administration of inhibitors of FGFR/AKT/ mTOR and cell cycle signaling could overcome EGFR-TKI resistance associated with shisa3-mediated CSC capacities in vivo.

Conclusion: Taken together, shisa3 was identified as a brake to EGFR-TKI resistance and CSC characteristics, probably through the FGFR/AKT/mTOR and cell cycle pathways, indicating that shisa3 and concomitant inhibition of its regulated signaling may be a promising therapeutic strategy for reversing EGFR-TKI resistance.

Keywords: Shisa3, Cancer stem cells, EGFR-TKI resistance, FGFR, mTOR

\section{Introduction}

EGFR tyrosine kinase inhibitors (EGFR-TKIs) have been an effective therapy for lung adenocarcinoma patients with activating mutations; however, therapeutic resistance to EGFR-TKIs inevitably develops [1]. Of note, cancer stem cells (CSCs), which can regrow after clinical

\footnotetext{
* Correspondence: nanwu@bjmu.edu.cn; zlyangyue@bjmu.edu.cn

† Jiahui Si and Yuanyuan Ma contributed equally to this work.

Department of Thoracic Surgery II, Key Laboratory of Carcinogenesis and Translational Research (Ministry of Education), Peking University Cancer Hospital and Institute, 52 Fucheng Road, Haidian District, Beijing 100142, People's Republic of China
}

management, play an important role in resistance to chemotherapy, targeted therapy and immunotherapy [2, 3]. Lung cancer CSCs can be identified by the surface markers CD133, CD44, ALDH1 and ABCG2 and are regulated by Notch, Wnt and cell cycle signaling pathways, which are critical for maintaining drug resistance [3]. Anti-CSC therapeutics targeting surface markers and associated pathways in different cancer types have been developed in animal models and investigated in clinical trials $[4,5]$. Thus, a comprehensive understanding of the molecular mechanism of CSC regulation in

(c) The Author(s). 2019 Open Access This article is distributed under the terms of the Creative Commons Attribution 4.0 International License (http://creativecommons.org/licenses/by/4.0/), which permits unrestricted use, distribution, and 
EGFR-TKI resistance might provide a novel strategy for treatment intervention.

The role of tumor suppressor genes in modulating the EGFR-TKI response has attracted the attention of researchers. FOXO3a, as a suppressor, has been shown to increase EGFR-TKI sensitivity and reduce CSC properties [6]. Shisa, as a tumor suppressor, has been discovered to antagonize the CSC-associated Wnt pathway and FGF signaling by interacting with immature forms of their receptors [7]. Sox2, an essential transcriptional factor of CSCs, was upregulated after FGFR1 activation [8], and FGFR1 signaling has been shown to contribute to the maintenance of CSC properties by interacting with the Hippo/YAP1 pathway in lung cancer [9]. Shisa3 has been reported to accelerate the degradation of $\beta$-catenin, a key component of Wnt signaling, which inhibits tumorigenesis, invasion and metastasis in lung cancer [10].

In addition to the acquisition of EGFR mutations, such as T790 M (resistance to gefitinib, erlotinib and ecotinib) [11] and C797S (resistance to osimertinib) [12], the activation of receptor tyrosine kinases (RTKs) combined with triggering downstream signaling pathways, including RAS and mitogen-activated protein kinase (MAPK), phosphoinositide 3-kinase (PI3K) and Akt, or signal transducer and activator of transcription 3 (STAT3), has been recognized to drive EGFR-TKI resistance in lung cancer [13]. Recently, it has been reported that the use of Akt inhibitor plus EGFR-TKIs led to suppressed growth in lung adenocarcinoma models of TKI resistance [14]. In the presence of mTOR activation, TKIresistant lung adenocarcinoma cells showed a better response to combinational treatment with mTOR and TKIs [15].

In the current study, we screened shisa3 as a tumor suppressor with decreased expression in lung adenocarcinoma patients with EGFR-TKI resistance. Intriguingly, shisa3 combined with gefitinib/osimertinib dramatically inhibited tumor growth of TKI-resistant lung adenocarcinomas. Shisa3 blockade drove EGFR-TKI resistance, cell cycle arrest and CSC enrichment by regulating the FGFR-dependent Akt/mTOR signaling pathway. Further studies indicated that TKIs combined with cell cycle and mTOR inhibitors significantly restrained tumor growth in TKI-resistant lung adenocarcinoma xenografts with upregulated shisa3 and downregulated Ki67. Taken together, targeting shisa3-regulated networks may provide a novel treatment strategy for reversing TKI drug resistance and CSC activities.

\section{Methods}

\section{Specimens}

In this study, all the samples were obtained from the lung adenocarcinoma patients who received surgery and neoadjuvant treatment before resection at Peking University Cancer Hospital (Beijing, China). All participants provided written informed consent, and the studies were approved by the Ethics Committee of Peking University Cancer Hospital. Paraffin tumor tissues from 45 cases of EGFR mutant stage III lung adenocarcinoma patients who had received EGFR-TKI treatment (gefitinib or icotinib) after surgery were investigated; these patients were enrolled from December 2009 to January 2013. Response to treatment was evaluated according to the Response Evaluation Criteria in Solid Tumors.

In all, 102 paraffin tumor tissue samples with EGFR mutation were collected from these lung adenocarcinoma patients who underwent surgery from January 2009 to November 2011. In addition, 38 pairs of frozen tumor tissues and normal tissues from the lung adenocarcinoma patients with EGFR mutation were involved in 2012. All patients were regularly followed, and the clinical outcomes of all the patients were obtained.

\section{Transcriptome sequencing (RNA-seq)}

Total mRNA was extracted using TRIzol reagent (\#15596018, Invitrogen, Carlsbad, CA, USA) according to the manufacturer's instructions. RNA-seq and subsequent data analyses were performed by the Beijing Institute of Genome Research, Chinese Academy of Sciences (Beijing, China). Briefly, $2 \mu \mathrm{g}$ RNA per sample was used to construct a cDNA library and was sequenced on the Illumina HiSeq 2500 with $125-150$ bp paired-end reads following the manufacturer's recommendations. Bowtie software was used to align the raw reads to the Homo sapiens genome sequences (NCBI). The false discovery rate (FDR, i.e., a probability of wrongly accepting a difference) of each gene was determined according to the Bonferroni correction method. Differential expression analysis was performed using the edge $\mathrm{R}$ package (2.6.2). An adjusted $P$-value $<0.05$ and FDRs $<0.01$ were set as the threshold for significantly differential expression.

\section{Quantitative real-time PCR (qRT-PCR)}

Total RNA was isolated from tissues and cells using TRIzol reagent (Invitrogen) following the manufacturer's instructions, and synthesis of cDNA from total RNA $(2 \mu \mathrm{g})$ was performed using a commercially available kit (EasyScript First-Strand cDNA Synthesis SuperMix, Transgen Biotech, Beijing, China). qRT-PCR was performed with the LightCycler 480 SYBR Green I Master using a LightCycler 480 Real-Time PCR System (Roche, Mannheim, Germany). The relative expression level of genes was normalized to GAPDH. The fold change was calculated according to $2^{-\Delta \mathrm{Ct}}$, in which $\Delta \mathrm{Ct}=\mathrm{Ct}$ target $\mathrm{Ct}$ control. All PCR assays were carried out in triplicate, 
and the mean of triplicates is reported. Primers are listed in Additional file 1: Table S1.

\section{Immunohistochemistry (IHC)}

Formalin-fixed and paraffin-embedded lung adenocarcinoma samples were incubated with primary anti-shisa3 (1:1000 dilution; Thermo Fisher Scientific, Rockford, IL, USA), anti-FGFR1 (1:200 dilution; Abcam, Cambridge, MA) or Ki-67 (1:200 dilution; ZSGB-BIO, Beijing, China)overnight at $4{ }^{\circ} \mathrm{C}$ followed by $\operatorname{IgG} / \mathrm{HRP}$ polymer (ZSGB-BIO) and diaminobenzidine substrate (ZSGB$\mathrm{BIO})$ in compliance with protocols. Staining results were independently evaluated by two experienced pathologists who were blinded to all clinical data.

\section{Cell lines and cell culture}

The human lung adenocarcinoma cell lines PC9, HCC827 and H1975 were maintained in our laboratory. Cell lines were cultured in RPMI-1640 medium (Gibco BRL, Gaithersburg, MD) supplemented with $10 \%$ fetal bovine serum (Gibco BRL), $100 \mathrm{U} / \mathrm{mL}$ penicillin, and $100 \mathrm{~g} / \mathrm{mL}$ streptomycin (Invitrogen, Grand Island, NY, USA) and incubated in a humidified incubator $\left(37^{\circ} \mathrm{C}\right)$ with $5 \% \mathrm{CO}_{2}$. All cell lines were certified by shorttandem repeat (STR) analysis.

\section{Reagents and antibodies}

Gefitinib (ZD1839), osimertinib (AZD9291), BGJ398, dactolisib (BEZ235) and PD0332991 were purchased from Selleck Chemicals (Houston, TX, USA). Reagents were formulated and stored according to the manufacturer's protocols. The following primary antibodies were used: AKT (\#9272), p-AKT S473 (\#9271), mTOR (\#2983), p-mTOR S2448 (\#2971), S6K (\#9205S), p-S6K Thr389 (\#9202S), S6 (\#2217), p-S6 Ser235/236 (\#4858), and FGFR3 (\#4574) from Cell Signaling Technology (Danvers, MA, USA); Shisa3 (\#167069, for Western blot), FGFR1 (\#ab10646) and p-FGFR3 Y724 (\#ab155960) from Abcam (Cambridge, MA); p-FGFR1 T653/T654 (\#06-1433) from Merck Millipore (Darmstadt, Germany), cyclin D1 (\#sc-8396), CDK4 (\#sc-23, 896), CDK6 (\#sc-7961), and p16 (\#sc-166,760) from Santa Cruz Biotechnology (Santa Cruz, CA); $\beta$-actin (\#HRP-60008) from Proteintech Group (Rosemont, IL, USA). The secondary HRP-conjugated goat anti-rabbit (\#CW0103S) and anti-mouse antibodies (\#CW0102S) were from CWBIO (Beijing, China).

\section{Generation of EGFR-TKI-resistant cells (PC9/ER)}

PC9/ER cells were developed from the parental PC9 cells by 6 months of exposure to gefitinib, starting at $10 \mathrm{nM}$ and increasing stepwise to $10 \mu \mathrm{M}$. At $80-90 \%$ confluence, the cells were detached with trypsin/EDTA (Gibco BRL) and divided into 2 parts. One part of the cells was frozen, and the other was reseeded into a new dish at doses $30-50 \%$ higher than the original. Control cells were treated parallel with vehicle (DMSO). The PC9/ER cells were validated to be resistant to gefitinib and osimertinib as shown in the results.

\section{Vector construction and transfection}

The lentiviral vector of shisa3 was constructed by inserting a shisa3 cDNA (NM_001080505.2) fragment into a lentiviral shuttle vector. Shisa3 knockdown was accomplished using specific shRNAs targeting shisa3. The shRNA sequences were as follows: shRNA-shisa3\#1: $5^{\prime}$ GTGGCTATTTATTGTTGCA-3'; shRNA-shisa3\#2: 5' GCTCCATCTTCATTGCGTT-3'; and shControl: 5' TTCTCCGAACGTGTCAGGT-3'. The packing and purification of the lentiviral vectors were performed by the GenePharma Company (Shanghai, China). The indicated cells infected with the lentiviral vectors were selected with puromycin for 2 weeks.

Tet-on expression plasmids were constructed by pELNS-M2rtTA-IRES-Neo and plenti6-TREpitt-GFP vectors for shisa3 expression.

pENTER-shisa3-flag was constructed by cloning the coding sequence (CDS) of shisa3 into the pENTER plasmid using the restriction sites Asis I and Mlu I. For further experiments, PC9/ER cells were transiently transfected with this plasmid using Lipofectamine ${ }^{\mathrm{Tw}} 3000$ (Invitrogen) according to the manufacturer's protocols.

\section{Western blot analysis}

The proteins of cells were extracted using RIPA buffer containing a complete protease inhibitor cocktail (Roche, Mannheim, Germany). Protein concentrations were measured with a bicinchoninic acid (BCA) protein assay kit (Beyotime). Equal amounts of protein were separated with $8 \%$ or $10 \%$ SDS-PAGE and transferred to polyvinylidene fluoride (PVDF) membranes. After blocking with $5 \%$ BSA (Amresco) or fat-free milk, the membranes were probed with primary antibodies at $4{ }^{\circ} \mathrm{C}$ overnight followed by secondary antibodies at room temperature for $1 \mathrm{~h}$. The proteins were then detected by chemiluminescence using Immobilon Western Chemiluminescent HRP Substrate (\#WBKLS0500, Millipore) and visualized using Amersham Imager 600 (GE Healthcare, Chicago, IL).

\section{Microarray and computational analysis}

PC9-shControl and PC9-shShisa3 cells were submitted to BoHao Bio-tech (Shanghai, China) for mRNA microarray analysis. Total RNA was purified with an RNeasy mini kit (Cat.\# 74,106, QIAGEN, GmBH, Germany) and hybridized using the Gene Expression Hybridization Kit (Cat.\# 5188-5242, Agilent Technologies, Santa Clara, CA, US). Data were extracted with Feature Extraction 
10.7 software (Agilent Technologies). Genes that were up- or downregulated with $>2$-fold change (FC) and a significant difference of $P<.05$ were further subjected to computational simulation by Ingenuity Pathway Analysis (IPA; QIAGEN, Valencia, CA, USA) online tools for an enrichment analysis.

\section{Cell viability}

A total of 3000-5000 cells were seeded per well in 96well plates and allowed to attach for $24 \mathrm{~h}$. Following treatment, cell viability was measured using a CCK-8 commercial kit (\#CK04, Dojindo, Japan) according to the manufacturer's protocol, and the absorbance at 450 $\mathrm{nm}$ was measured using a spectrophotometer. The cell half maximal inhibitory concentration (IC50) was calculated using GraphPad software.

\section{Sphere formation assay}

The cells were trypsinized and washed in phosphatebuffered saline (PBS), and single cells were plated in ultralow-attachment 96-well plates (Corning Inc., Life Sciences). DMEM/F-12 (Invitrogen) serum-free medium including $20 \mathrm{ng} / \mathrm{mL}$ basic fibroblast growth factor (Peprotech, Rocky Hill, NJ, USA), 20 ng/mL epidermal growth factor (Peprotech), B27 (Invitrogen), $10 \mathrm{ng} / \mathrm{mL}$ hepatocyte growth factor (Peprotech), and 1\% methylcellulose (Sigma, MO, USA) was used to cultivate the cells for 12 days. The spheres were counted under a light microscope. Images are shown as representatives of three independent experiments. Sphere formation efficiency was calculated as follows: sphere formation efficiency $=$ sphere/input cells $\times 100 \%$.

\section{Migration and invasion assays}

A total of $1.0 \times 10^{5}$ cells per well in serum-free RPMI were placed in the upper chamber (Cat NO. 3422, Corning Costar, Cambridge, MA) with/without precoated Matrigel (Cat NO. 356234, BD Biosciences, San Jose, California, USA) following the manufacturer's instructions. The lower chambers were filled with culture medium supplemented with $10 \%$ FBS. The indicated cells were allowed to migrate or invade through pores for 12 to $24 \mathrm{~h}$ at $37^{\circ} \mathrm{C}$. The total numbers of migrated or invaded cells in the lower chambers were fixed in paraformaldehyde (4\%) and stained with $0.1 \%$ crystal violet for $5 \mathrm{~min}$ at room temperature and counted under a microscope.

\section{Cell cycle assay}

The effect of shisa3 on cell cycle distribution was analyzed by flow cytometry. After being starved overnight and stimulated with complete medium for $24 \mathrm{~h}$, cells were collected, washed with PBS, and fixed in $75 \%$ immediately precooled ethanol overnight at $-20^{\circ} \mathrm{C}$. After washing with PBS, cells were stained with propidium iodide (PI)/RNase (BD Biosciences) at room temperature for $15 \mathrm{~min}$ in the dark and analyzed by flow cytometry within $1 \mathrm{~h}$. Quantitative cell cycle analysis was assessed with ModFit version 3.0 software (Verity Software House, Topsham, ME).

\section{Xenografts and treatments}

Nonobese diabetic/severe combined immunodeficiency (nod-scid) mice were cared for in accordance with guidelines approved by the Ethics Committee of Animal Experiments of Peking University Cancer Hospital. A total of $3 \times 10^{6}$ cells were subcutaneously injected into the right flank of 6-week-old female nod-scid mice (Beijing HFK Bioscience Co., Ltd., China). When tumors reached a size of approximately $100 \mathrm{~mm}^{3}$, the mice were randomized into several groups separately via oral gavage with different treatments. Tumors were measured every 4 days using calipers, and tumor volume was calculated using the formula volume $=\left(\right.$ length $\times$ width $\left.^{2}\right) / 2$. At the end of the treatments, the mice were sacrificed with $\mathrm{CO}_{2}$, and the tumors were stripped for successive assays.

\section{Immunoprecipitation}

Cells were washed twice in ice-cold PBS, harvested and lysed with RIPA lysis buffer (\#P0013D, Beyotime, Wuhan, China) for immunoprecipitation experiments. For each sample, $1.5 \mathrm{mg}$ of protein was incubated with Protein G Sepharose 4 Fast Flow (\#17061802, GE Healthcare, UK) and anti-Flag antibody (\#66008-2-Ig, Proteintech) or anti-IgG antibody (\#A7028, Beyotime). After an overnight incubation at $4{ }^{\circ} \mathrm{C}$, the beads were washed, and the final pellet was suspended in RIPA buffer. Bound proteins were eluted from the beads by heating and centrifugation and then analyzed by Western blot.

\section{Statistical analysis}

The relationship between the expression of shisa 3 and patients' clinical variables was assessed using the $X^{2}$-test and Fisher's exact test. The disease-free survival (DFS) and overall survival (OS) of patients were estimated using the Kaplan-Meier method and the log-rank test. The Cox hazard proportional model was applied to multivariate analysis. All statistical analyses were performed using SPSS version 20.0 software (SPSS Inc., Chicago, IL), and images were plotted using GraphPad Prism 7 (GraphPad Software, La Jolla, CA, USA). Differences were analyzed using unpaired two-tailed $t$ tests. All data are representative of 3 independent experiments and are illustrated as the means \pm SDs. $(P<0.05$ was considered statistically significant.) 


\section{Results}

Decreased shisa3 is associated with EGFR-TKI resistance in lung adenocarcinoma

We used RNA-seq to screen gene expression levels in local advanced lung adenocarcinoma patients who received EGFR-TKI (gefitinib or ecotinib) therapy (Additional file 1; Table S2). There were 3 drugsensitive patients with partial response (PR) and 3 drug-resistant patients with stable disease (SD). Herein, we found that 5 genes (Shisa3, PADI1, LRP2, ANGPTL4 and ALOX15B) were upregulated and 4 genes (CXCL9, ADAMDEC1, SCGB1A1/CC10, HLAG) were downregulated in $P R$ tumor tissues compared to SD tumor tissues (Fig. 1a). Owing to the genes that could inhibit EGFR-TKI resistance, we focused on shisa3, which was the most upregulated gene in PR samples with a 4.68-fold increase. In addition, shisa3 was validated to be highly expressed in normal tissues compared to paired tumor tissues of lung adenocarcinoma with EGFR activating mutations (Fig. 1b, $n=38$ ).
We further determined by IHC detection whether shisa3 was associated with the therapeutic effect of EGFR-TKIs in lung adenocarcinoma patients with EGFR mutations $(n=45)$ who received gefitinib/ecotinib treatment. Based on the divided groups of low shisa3 expression $(-/+)$ and high shisa3 expression $(++/+++)$ (Fig. 1c), an increased rate of high expression was observed in EGFR-TKI-sensitive patients (PR: 75\%) compared to EGFR-TKI-resistant patients (SD: 31\%) (Fig. 1d). Thus, we demonstrated that patients with high expression of shisa3 have a better response to EGFR-TKIs, indicating that shisa3 may be used to predict the efficacy of TKI therapy in lung adenocarcinoma patients.

A previous study of 69 samples from non-small-cell lung cancer (NSCLC) patients revealed that shisa3 was positively correlated with better prognosis [10]. Subsequently, we investigated shisa3 status in 102 tissue samples from lung adenocarcinoma patients with EGFR mutations. There was no significant difference between shisa3 expression level and sex, age, smoking

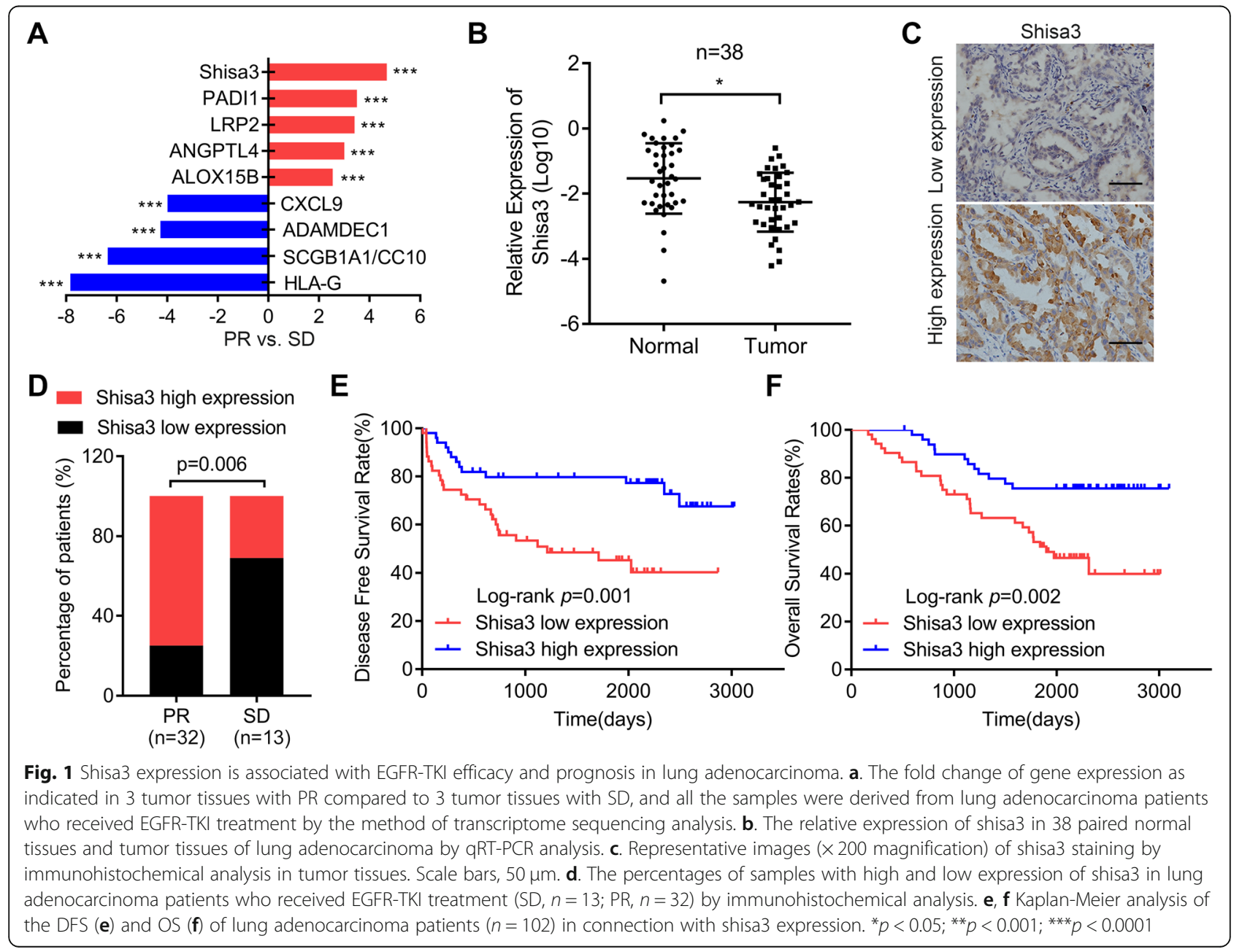


history, venous invasion, and differentiation (Table 1). We found that low expression of shisa 3 was related to later TNM stage and lymph node metastasis. We further obtained evidence that low expression of shisa3 was associated with shorter DFS and OS (Fig. 1e-f). Shisa3 was identified as an independent OS factor for lung adenocarcinoma by univariate and multivariate Cox regression analyses (Table 2).

These data suggested that shisa3 may drive sensitivity to EGFR-TKIs in EGFR-mutant lung adenocarcinoma.

\section{The established EGFR-TKI-resistant cells induced the CSC phenotype}

Consistent with previous studies [16-18], we verified that PC9 (gefitinib IC50 $=0.017 \pm 0.003 \mu \mathrm{M}$, osimertinib IC50 $=0.013 \pm 0.012 \mu \mathrm{M}$ ) and HCC827 (gefitinib IC50 = $0.013 \pm 0.006 \mu \mathrm{M}$, osimertinib IC50 $=0.002 \pm 0.001 \mu \mathrm{M})$ cells were sensitive to EGFR-TKIs and that H1975 (gefitinib IC50 $=23.64 \pm 1.42 \mu \mathrm{M}$, osimertinib IC50 $=0.094 \pm$ $0.011 \mu \mathrm{M})$ cells were resistant to a first-generation EGFR-TKI (gefitinib) but sensitive to a third-generation EGFR-TKI (osimertinib) (Fig. 2a-b). Next, we generated EGFR-TKI-resistant PC9/ER cells derived from PC9

Table 1 Clinicopathological variables and shisa3 expression in lung adenocarcinoma patients $(n=102)$

\begin{tabular}{|c|c|c|c|c|}
\hline \multirow[t]{2}{*}{ Variable } & \multirow{2}{*}{$\begin{array}{l}\text { Case no. } \\
(\%)\end{array}$} & \multicolumn{2}{|l|}{ Shisa3 } & \multirow{2}{*}{$\begin{array}{l}P \\
\text { value }\end{array}$} \\
\hline & & Low expression & High expression & \\
\hline Gender & & & & 0.712 \\
\hline Male & $43(42.2 \%)$ & $21(48.8 \%)$ & $22(51.2 \%)$ & \\
\hline Female & $59(57.8 \%)$ & 31 (52.5\%) & $28(47.5 \%)$ & \\
\hline Age (years) & & & & 0.436 \\
\hline$\leq 60$ & 47 (46.1\%) & $22(46.8 \%)$ & $25(53.2 \%)$ & \\
\hline$>60$ & 55 (53.9\%) & $30(54.5 \%)$ & $25(45.5 \%)$ & \\
\hline Smoking history & & & & 0.874 \\
\hline Yes & $62(60.8 \%)$ & $32(51.6 \%)$ & $30(48.4 \%)$ & \\
\hline No & $40(39.2 \%)$ & $20(50.0 \%)$ & $20(50.0 \%)$ & \\
\hline Venous invasion & & & & 0.390 \\
\hline Negative & $80(78.4 \%)$ & 39 (48.8\%) & $41(51.2 \%)$ & \\
\hline Positive & $22(21.6 \%)$ & $13(59.1 \%)$ & $9(40.9 \%)$ & \\
\hline Differentiation & & & & 0.789 \\
\hline Poor & $36(35.3 \%)$ & 19 (52.8\%) & $17(47.2 \%)$ & \\
\hline Moderate/well & $66(64.7 \%)$ & $33(50.0 \%)$ & $33(50.0 \%)$ & \\
\hline TNM stage & & & & 0.098 \\
\hline$|/| \mid$ & 74 (72.5\%) & 34 (45.9\%) & 40 (54.1\%) & \\
\hline III & $28(27.5 \%)$ & $18(64.3 \%)$ & $10(35.7 \%)$ & \\
\hline N stage & & & & 0.013 \\
\hline 0 & $63(61.8 \%)$ & $26(41.3 \%)$ & 37 (58.7\%) & \\
\hline $1 / 2$ & 39 (38.2\%) & $26(66.7 \%)$ & $13(33.3 \%)$ & \\
\hline
\end{tabular}

Bold values are significant $(p<0.05)$ cells, showing a 1315.6-fold increase in IC50 for gefitinib and a 196.3-fold increase in IC50 for osimertinib. In addition, compared with HCC827 cells, PC9/ER cells demonstrated a 1698.8-fold increase in gefitinib IC50; compared with HCC827 cells, PC9/ER cells exhibited a 1429.0-fold increase in osimertinib IC50. Among the EGFR hotspot analyses, only a sensitive deletion mutation of Exon 19 was identified in PC9/ER cells (Additional file 1; Table S3). In view of the decreased expression of shisa3 in lung adenocarcinoma tissues that were resistant to EGFR-TKI treatment, we detected this gene expression in lung adenocarcinoma cells with variable IC50 to gefitinib/osimertinib. Lower expression of shisa3 was detected in PC9/ER cells compared to PC9, HCC827 and H1975 cells (Fig. 2c).

Considering that tumor cells with EGFR-TKI resistance may manifest stem-cell-like properties $[19,20]$, we then analyzed whether PC9/ER cells exhibited a CSC phenotype. The primary and secondary sphere formation efficiencies were elevated in PC9/ER cells compared with PC9 cells (Additional file 1: Figure S1A-B). The expression levels of CSC-related factors, including Sox2, Oct4, Nanog and ABCG2, were increased in PC9/ER cells (Additional file 1: Figure S1C). Migration and invasion properties were enhanced in PC9/ER cells compared to PC9 cells (Additional file 1: Figure S1D-E). We then performed a limited dilution assay by transplanting PC9 and PC9/ER cells into nod-scid mice. PC9/ER cells, which showed increased tumorigenic frequency, formed more and larger tumors than PC9 cells (Additional file 1: Figure S1F). Based on the reported mechanisms in EGFR-TKIs resistance [21], we then investigated Met, HER-2, PTEN and EMT related signaling in PC9/ER and the parental PC9 cells. Among those factors, Met expression level and EMT activity (decrease of Ecadherin, and increase of $\mathrm{N}$-cadherin and vimentin) were enhanced in the PC9/ER cells (Additional file 1: Figure S1G). In addition, the CSC related makers including CD133, CD44 and ALDH1A1 were up-regulated in the PC9/ER cells, compared to the parental PC9 cells (Additional file 1: Figure S1H).

These results suggested that PC9/ER cells with decreased expression of shisa3 demonstrated dramatic resistance to EGFR-TKIs and showed an enhanced CSC phenotype.

Overexpressed shisa3 attenuates EGFR-TKI resistance and suppresses the CSC phenotype

To investigate the biological effect of shisa3, we overexpressed and validated the increased expression of this protein in PC9/ER and H1975 cells (Additional file 1: Figure S2A). Shisa3 resulted in a decreased proliferation in PC9/ER cells (Additional file 1: Figure S2B) and H1975 cells (Additional file 1: Figure S2C). Since shisa3 as a tumor suppressor gene, shisa3 was established in 
Table 2 Univariate and multivariate cox regression analyses for overall survival (OS) in lung adenocarcinoma patients ( $n=102)$

\begin{tabular}{|c|c|c|c|c|}
\hline \multirow[t]{2}{*}{ Variables } & \multicolumn{2}{|l|}{ Univariate analysis } & \multicolumn{2}{|l|}{ Multivariate analysis } \\
\hline & $\mathrm{HR}(95 \% \mathrm{Cl})$ & $P$ value & $\mathrm{HR}(95 \% \mathrm{Cl})$ & $P$ value \\
\hline Gender & $0.593(0.319-1.103)$ & 0.099 & NA & \\
\hline Age & $1.036(0.553-1.914)$ & 0.911 & NA & \\
\hline Smoking history & $1.522(0.818-2.831)$ & 0.185 & NA & \\
\hline Venous invasion & $1.848(0.939-3.636)$ & 0.075 & NA & \\
\hline Differentiation & $0.572(0.307-1.068)$ & 0.079 & NA & \\
\hline TNM stage & $2.649(1.413-4.968)$ & 0.002 & $3.452(1.009-11.814)$ & 0.048 \\
\hline N stage & $1.946(1.046-3.621)$ & 0.035 & NA & \\
\hline Shisa3 & $2.802(1.421-5.527)$ & 0.003 & $2.606(1.298-5.235)$ & 0.007 \\
\hline
\end{tabular}

$H R$ hazard ratio, $\mathrm{Cl}$ confidence interval, bold values are significant $(p<0.05)$

the Tet-on inducible system to transfect the PC9/ER and H1975 cells. The expression of shisa3 was significantly up-regulated after doxycycline induction for $48 \mathrm{~h}$ (Fig. 2d, and Additional file 1: Figure S2D). Shisa3 led to a decreased IC50 for gefitinib and osimertinib in PC9/ER cells (Fig. 2e). Moreover, inhibitory effect of gefitinib was enhanced in the H1975 cells overexpressing shisa3 induced by doxycycline (Additional file 1: Figure S2E).

We then examined whether shisa3 might be a suppressor that depresses the CSC phenotype. We observed a significant decrease in primary and secondary sphere formation efficiencies in PC9/ER cells overexpressing shisa3 (Fig. 2f-g). Lower expression levels of Sox2, Oct4, Nanog and ABCG2 were observed in shisa3overexpressing PC9/ER cells than in PC9/ER-control cells (Fig. 2h). CD133, CD44 and ALDH1A1 were downregulated in the shisa3-overexpression PC9/ER cells (Additional file 1: Figure S2F). When shisa3 was overexpressed in PC9/ER cells, fewer cells exhibited migration and invasion capacities (Additional file 1: Figure S2G, Fig. 2i). We then performed a limited dilution assay in nod-scid mice, showing fewer and smaller tumors and lower tumorigenic frequencies in shisa3-overexpressing PC9/ER cells (Fig. 2j).

\section{Knockdown of shisa3 triggers EGFR-TKI resistance and enhances the CSC phenotype}

Next, we downregulated shisa3 in PC9 and HCC827 cells by transfecting these cells with lentiviral-based shRNAs. The shisa3 expression level was validated to be suppressed by Shshisa3\#1 and Shshisa3\#2 (Fig. 3a). Decreased shisa 3 led to a 4.48 -fold increase in gefitinib IC50 and an 11.75-fold increase in osimertinib IC50 in PC9 cells (Fig. 3b). Knockdown of shisa3 increased cell viabilities in $\mathrm{HCC} 827$ cells treated with gefitinib and osimertinib (Fig. 3c).

Then, we analyzed how CSC properties changed in PC9 and HCC827 cells after shisa3 knockdown. It was shown that decreased expression of shisa3 enhanced the primary and secondary sphere formation efficiencies (Fig. 3d-e). Sox2, Oct4, Nanog and ABCG2 expression levels were higher in the PC9 cells transfected with Shshisa3\#1 than in the cells transfected with shControl (Fig. 3f). CD133, CD44 and ALDH1A1 were also induced in the PC9 cells with knock-down of shisa3 (Additional file 1: Figure S2H). The results showed more migratory and invasive cells in PC9 and HCC827 cells with suppressed shisa3 (Fig. 3g-i, Additional file 1: Figure S2I).

The above data indicated that shisa3 functions to reverse EGFR-TKI resistance and attenuate CSC potential.

Shisa3 interacts with FGFR to impact EGFR-TKI sensitivity Shisa has been reported to physically interact with FGFR and inhibit its protein maturation [7]; therefore, we explored whether shisa3 interacted with FGFR to modulate the EGFR-TKI response. We further verified the bands of FGFR1 and FGFR3 in immunoprecipitation by shisa3Flag in PC9/ER cells (Fig. 4a). We observed that overexpression of shisa3 dramatically reduced FGFR1, FGFR3, phosphorylated (p) FGFR1 (p-FGFR1) and p-FGFR3 expression levels in PC9/ER cells, and suppression of this gene induced these two receptors and their phosphorylation in PC9 cells (Fig. 4b). IHC was performed to identify FGFR1 expression in lung adenocarcinoma (Fig. 4c). A negative relationship was shown in the lung adenocarcinoma tissues with EGFR mutations ( $n=102$, Fig. $4 d$ ). In those patients, higher expression of FGFR1 was associated with shorter DFS and OS ( $n=102$, Fig. 4e-f). Then, the FGFR1/3 inhibitor-BGJ398 was used to treat the PC9/ER cells. In presence of shisa3, BGJ398 increased response to gefitinib and osimertinib by 21.63 and $25.87 \%$ in the PC9/ER cells, respectively (Fig. 4g).

These results indicated that shisa3 interacted with FGFR1/3 and inhibit their activation to increase the EGFR-TKI response to a certain extent. 


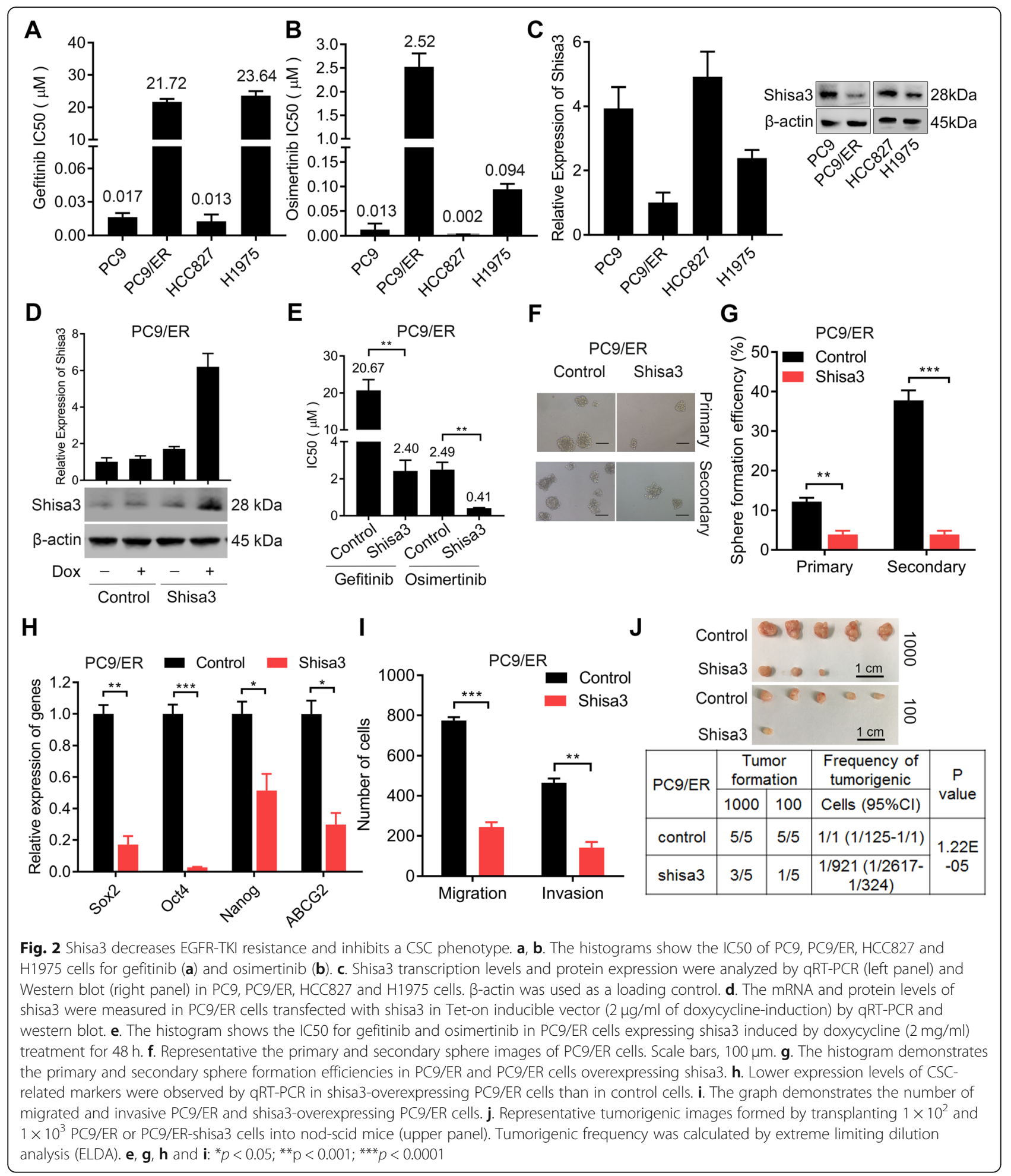

Shisa3 controls EGFR-TKI resistance by inhibiting FGFR/ AKT/mTOR and cell cycle signaling

To dissect the molecular mechanism of the shisa3mediated EGFR-TKI response, we performed microarray of RNA analysis in knocked-down shisa3 PC9 cells with 449 upregulated genes and 189 downregulated genes (Fig. 5a). Signaling pathways, including mTOR, cell cycle, FoxO, apoptosis, insulin and AMPK, were enriched when shisa3 was suppressed (Fig. 5b). 


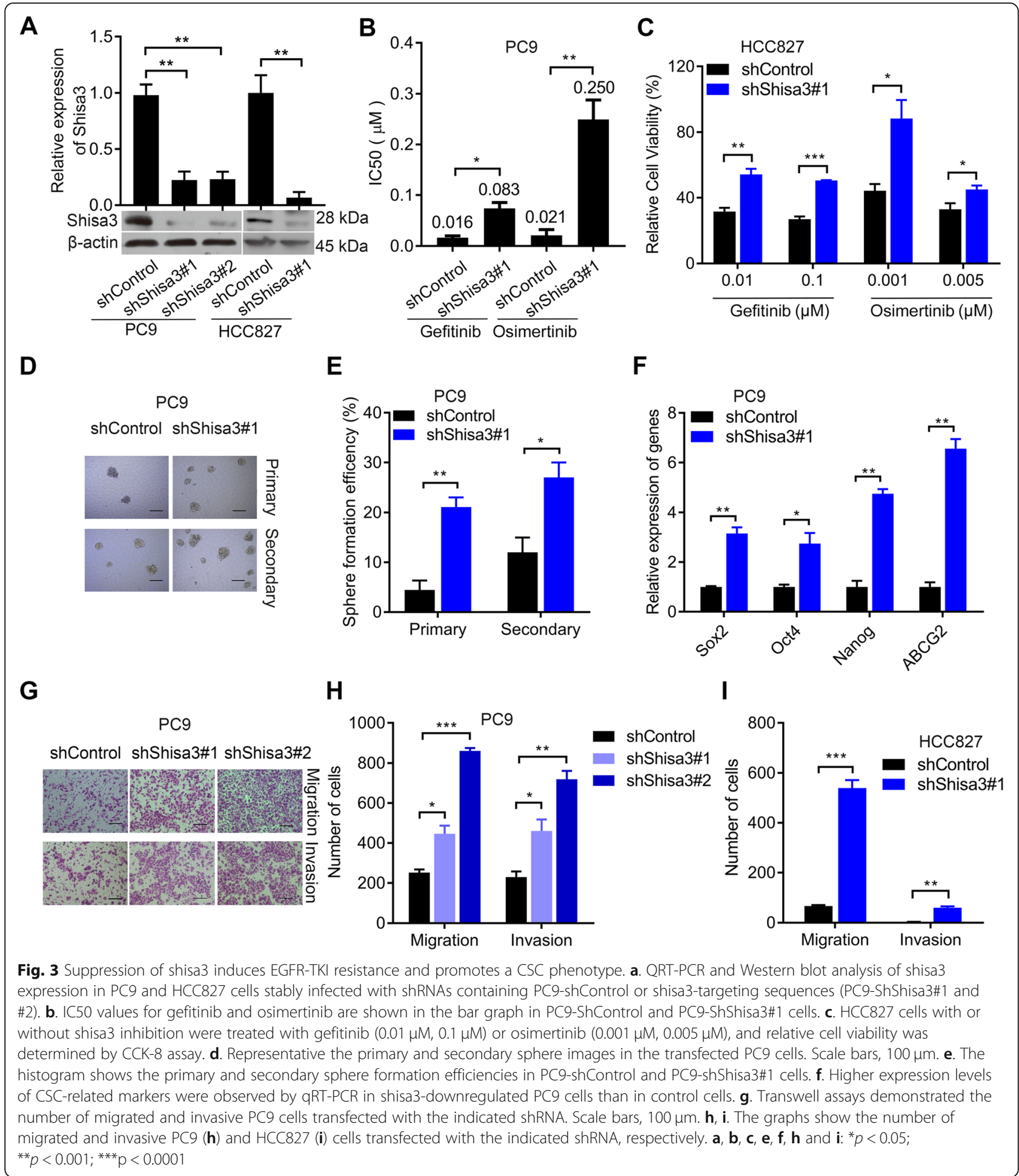

The increased genes associated with mTOR signaling were listed in the Additional file 1; Table S4. Furthermore, overexpression of shisa3 resulted in significant inhibition of p-AKT, p-mTOR, p-S6K and p-S6 in PC9/ER cells (Fig. 5c, left panel). In contrast, the loss of shisa3 increased the activation of p-AKT, p-mTOR, p-S6K and
p-S6 in PC9 cells (Fig. 5c, right panel). Then, we investigated whether a mTOR signaling inhibitor (BEZ235) could influence EGFR-TKI sensitivity in lung adenocarcinoma cells. Gefitinib, osimertinib, BEZ235 or a combination of gefitinib or osimertinib and BEZ235 suppressed PC9/ER cells by $17.82 \pm 10.06 \%, 9.49 \pm$ 


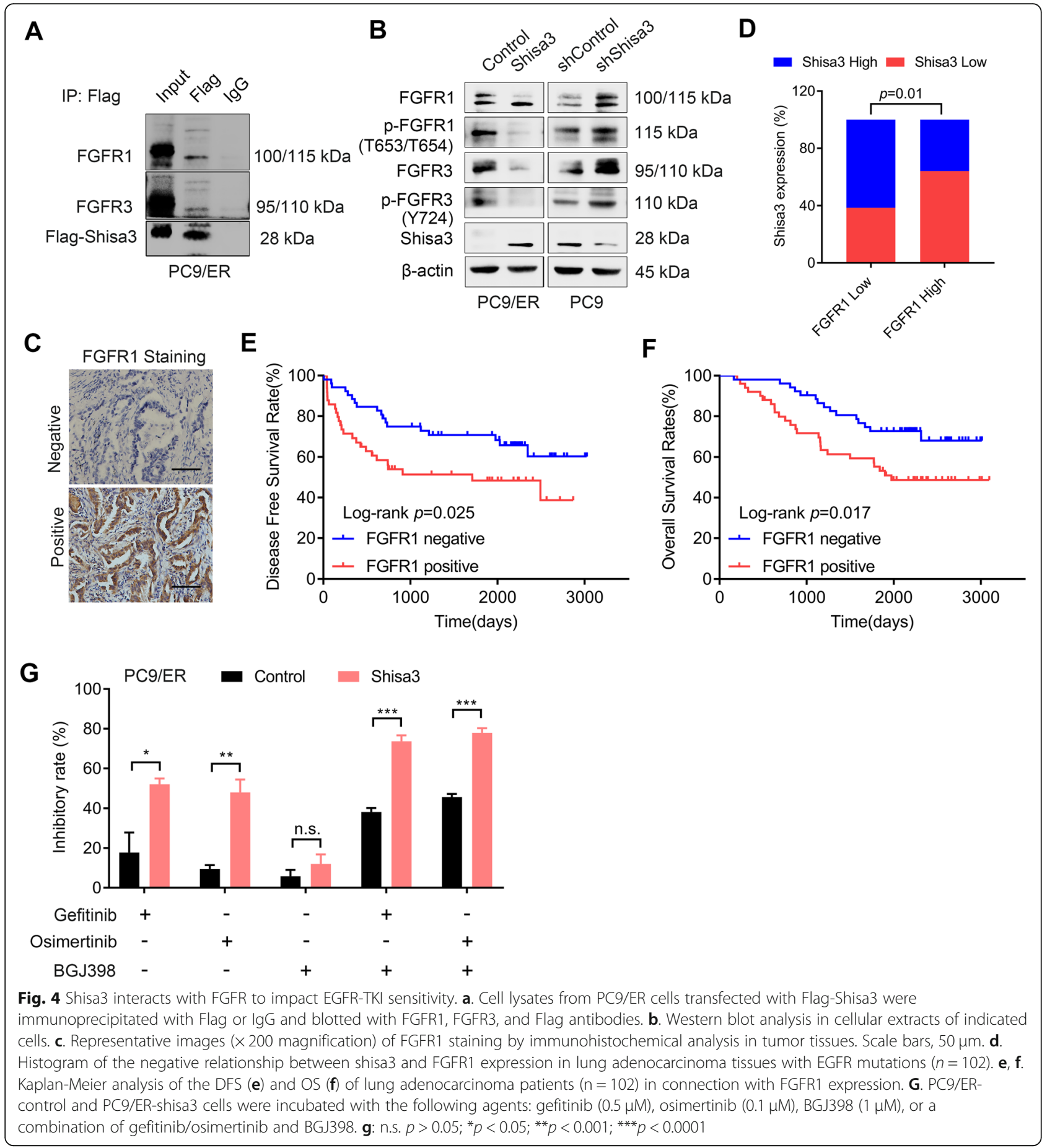

$11.84 \%, \quad 0.97 \pm 0.36 \%, \quad 45.15 \pm 3.19 \%$ or $50.21 \pm 1.32 \%$, and this inhibitory effect could be enhanced to $57.41 \pm$ $2.9 \%, \quad 47.97 \pm 6.48 \%, \quad 43.75 \pm 2.2 \%, \quad 67.09 \pm 3.92 \%$ or $85.56 \pm 2.6 \%$ in PC9/ER cells overexpressing shisa3, respectively (Fig. 5d).

Based on the microarray data (Fig. 5b, Additional file 1; Table S5), we validated whether shisa3 regulates cell cycle distribution. Cell cycle arrest was observed in the shisa3-overexpressing PC9/ER cells with increased G0/ G1 stage and decreased $S$ and G2/M stages (Fig. 5e). In contrast, suppression of shisa3 promoted the cell cycle in PC9 cells (Fig. 5f). In addition, lower expression of cyclin D1, CDK4 and CDK6 was observed in the shisa3overexpressing PC9/ER cells, and higher expression of these proteins was observed in PC9 cells with suppressed shisa3 (Fig. 5g). We then detected how the cell cycle 


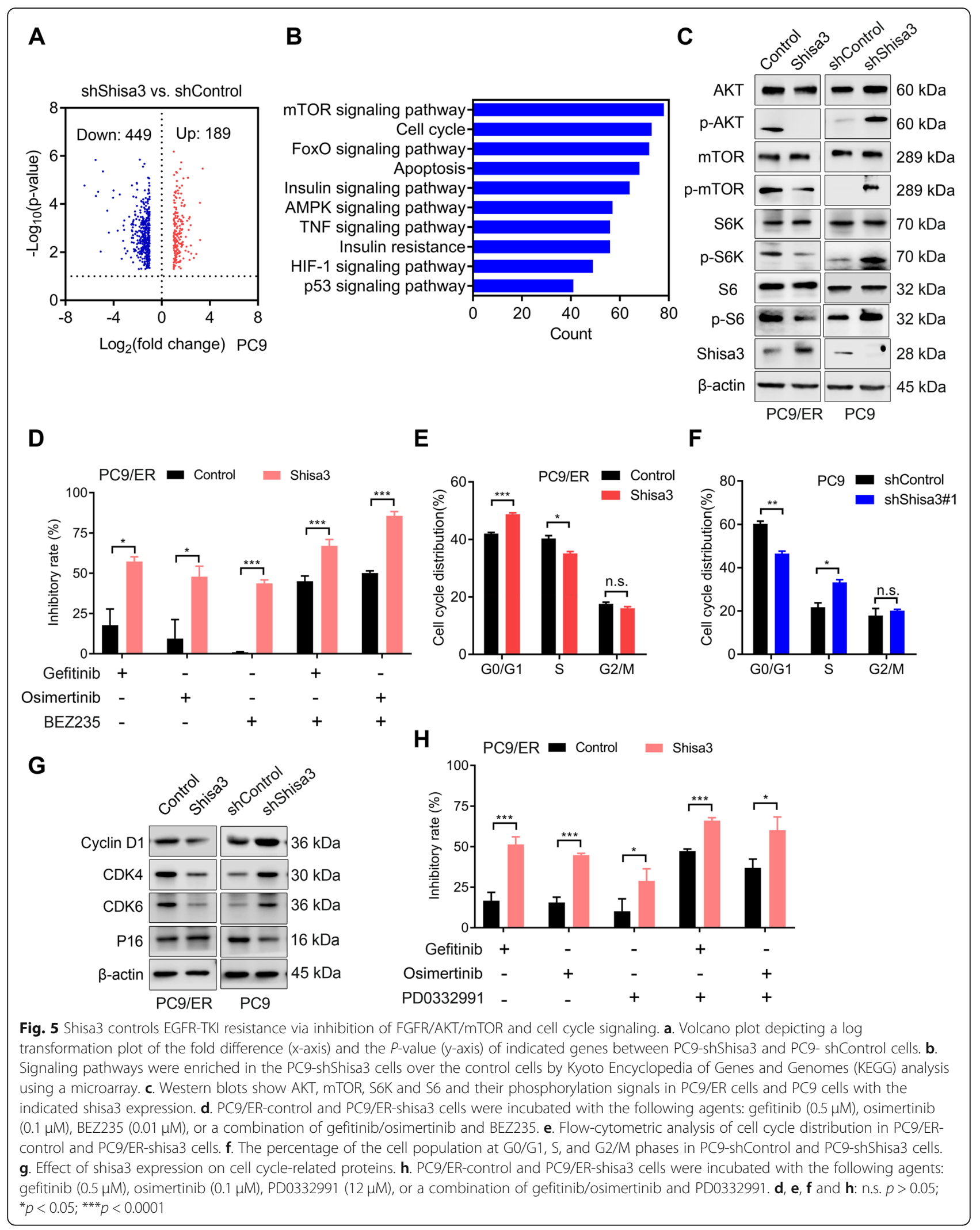


inhibitor palbociclib (PD0332991) influenced EGFR-TKI sensitivity. PD0332991 increased the inhibitory rate of gefitinib/osimertinib in PC9/ER cells overexpressing shisa3 (Fig. 5h).

AKT activation has been reported to mediate FGFR inhibitor resistance [22]. These data suggested that the shisa3-mediated increased response to EGFR-TKIs might be associated with the inactivation of FGFR/AKT/ mTOR and cell cycle signaling.

\section{Targeting shisa3-regulated signaling attenuated EGFR-TKI} resistance

Firstly, we tested inhibitory effect of gefitinib $(15 \mathrm{mg} / \mathrm{kg} /$ day) and osimertinib $(5 \mathrm{mg} / \mathrm{kg} /$ day $)$ on the formed tumors of PC9 cells. As shown in the Additional file 1: Figure S3A-C, gefitinib or osimertinib could significantly control tumor growth of PC9 cells, with 89 and 99\% inhibitory effect, respectively. To assess the role of shisa3 in inhibiting tumor growth of lung adenocarcinoma cells with EGFR-TKI resistance, we injected PC9/ER-control and PC9/ER-shisa3 cells into nod-scid mice. Until the tumor volume reached $100 \mathrm{~mm}^{3}$, the mice were subsequently treated with vehicle (1\% Tween 80 in PBS) as a control group, gefitinib $(60 \mathrm{mg} / \mathrm{kg} / \mathrm{d})$ or osimertinib $(25$ $\mathrm{mg} / \mathrm{kg} / \mathrm{d}$ ) by oral gavage for 14 days. Compared to the control group, the groups treated with gefitinib, osimertinib or shisa3 overexpression alone showed inhibited tumor growth by $55.23,67.20$ and $57.91 \%$, respectively, and the combination of gefitinib or osimertinib and shisa3 overexpression even dramatically suppressed tumor growth by 87.70 and $87.16 \%$, respectively (Fig. 6ab). Lower tumor weights were detected in the shisa3overexpressing PC9/ER tumors or the PC9/ER tumors with EGFR-TKI (gefitinib/osimertinib) treatment, and even the lowest tumor weights were observed in the shisa3-overexpressing PC9/ER tumors with gefitinib or osimertinib treatment (Fig. 6c).

Based on the above data, we further studied the therapeutic effect via targeting shisa3-regulated signaling, and the control group was showed as Fig. 6a-c. Compared to

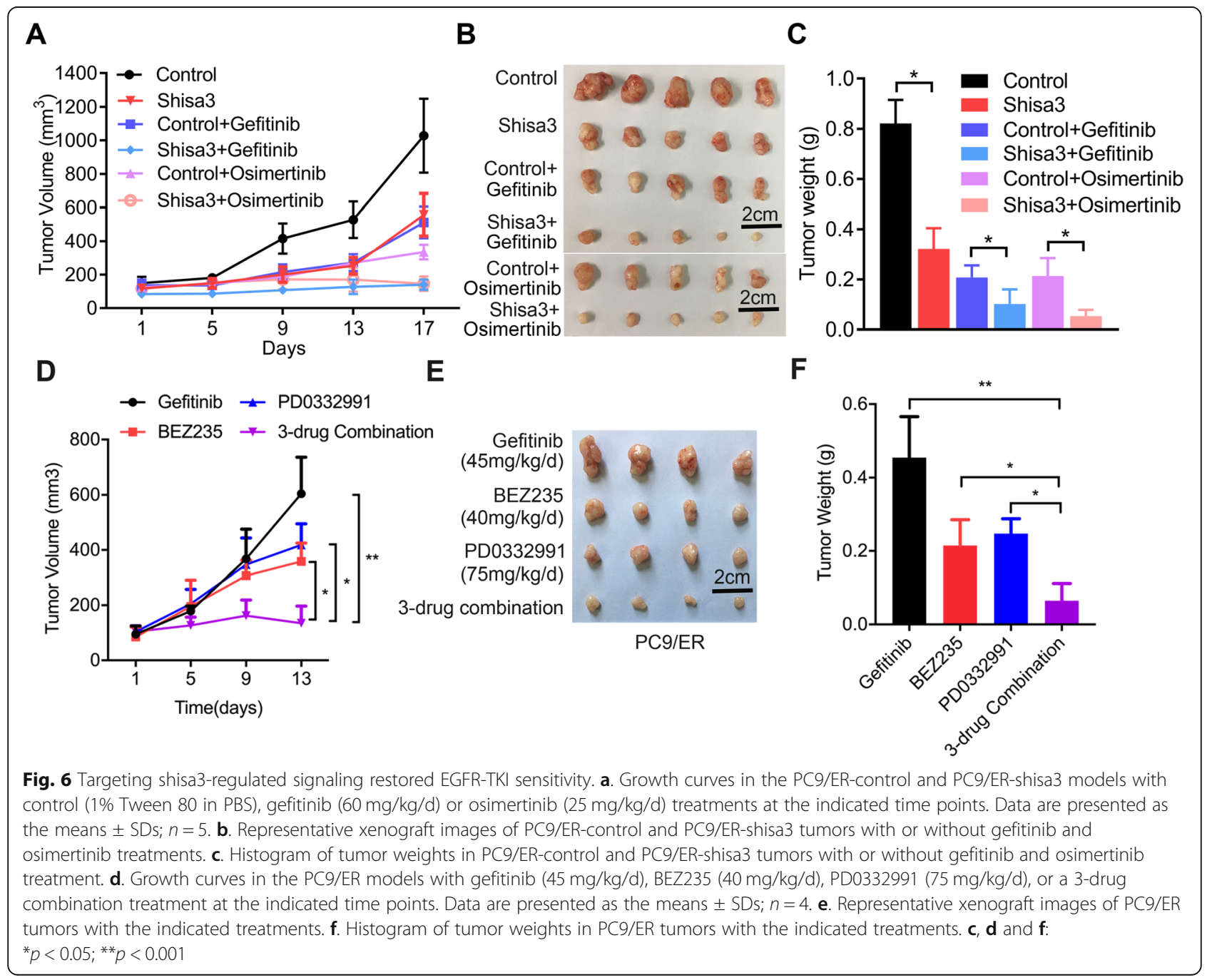


gefitinib treatment, BEZ235 or PD0332991 could inhibit tumor growth, and the combination of gefitinib, BEZ235 and PD0332991 even dramatically suppressed tumor growth in the EGFR-TKI resistant xenografts of PC9/ER (Fig. 6d-f). These data indicated that shisa3-regulated signaling may be a brake for lung adenocarcinoma with EGFR-TKI resistance.

Taken together, targeting shisa3-regulated signaling had an attenuated effect on EGFR-TKI-resistance that was associated with the depression of CSC properties (Fig. 7).

\section{Discussion}

Although EGFR-TKIs benefit lung cancer patients with sensitive mutations, most patients eventually develop drug resistance and relapse. Previously, the role of CSCs in EGFR-TKI resistance had not been well clarified. Deep study of the molecular mechanisms of the regulating network in EGFR-TKI resistance is critical, as it may promote the development of novel therapeutic strategies to overcome a failed treatment. In the current study, we screened and verified that shisa3, as a suppressor, prevents EGFRTKI resistance and suppresses the CSC phenotype in lung adenocarcinoma as follows: (1) Lung adenocarcinoma patients with high expression of shisa3 had a better response to EGFR-TKIs, indicating that shisa3 may be used to predict the efficacy of TKI therapy. (2) Shisa3 significantly suppressed self-renewal; expression levels of CSC-related factors; and migratory, invasive and tumorigenic capacities of CSC phenotypes, which can drive drug resistance in tumor cells. (3) Ectopic expression of shisa3 in vivo combined with gefitinib/osimertinib dramatically inhibited xenograft tumors from EGFR-TKI-resistant tumor cells. (4) Shisa3 altered the response of lung adenocarcinoma cells to EGFR-TKI treatment via FGFR/AKT/mTOR and cell cycle signaling. (5) TKIs combined with inhibitors of shisa3regulated downstream signaling had enhanced function to restrain tumor growth in gefitinib/osimertinib-resistant xenografts, suggesting a potential therapeutic strategy to reverse EGFR-TKI resistance.
Increasing evidence has shown that acquired resistance to EGFR-TKIs is associated with an improved CSC phenotype $[4,23]$. Herein, we found decreased shisa3 in gefitinib-resistant lung adenocarcinoma patients and EGFR-TKI-resistant PC9/ER cells. Consequently, shisa3 overexpression significantly controlled tumors derived from PC9/ER cells and CSC phenotypes. Shisa3 has been reported to accelerate the degradation of $\beta$-catenin in the Wnt signaling pathway, which regulates CSC maintenance in diverse types of cancer [24, 25]. Importantly, shisa3 combined with gefitinib and osimertinib inhibited tumor growth in PC9/ER xenografts, suggesting a potential role for this gene in reversing EGFR-TKI resistance.

Shisa3, located on chromosome 4p13, is a member of the shisa family, which mediates both WNT and FGF signaling by inhibiting the posttranslational maturation and cell surface trafficking of their receptors to cell surface [7]. Based on the above evidence showing the interaction of shisa with immature forms of FGFRs, we confirmed that ectopic shisa3 was immunoprecipitated with endogenous FGFR1 and FGFR3 in PC9/ER cells, indicating an interaction between shisa 3 and FGFR $1 / 3$ that is involved in EGFR-TKI resistance. The FGF2-mediated FGFR/ERK pathway was previously considered to regulate CSCs, and inhibition of this signaling could delay tumor growth in esophageal squamous cell carcinoma [26]. Thus, it is reasonable to speculate that shisa 3 decreased CSC characteristics by inhibiting the FGFrelated axis. As we expected, shisa3 restored sensitivity to gefitinib/osimertinib and decreased CSC characteristics linked to FGFR1/3 activity.

Multiple mechanisms of EGFR-dependent and EGFRindependent resistance have been described. It is known that RTKs such as EGFR and FGFR mostly result in activation of downstream MAPK or PI3K/AKT/mTOR pathways. Drug resistance could be caused by the PI3K/ $\mathrm{AKT} / \mathrm{mTOR}$ signaling pathway, which is associated with CSC sustainability [27, 28]. Aberrant activation of the AKT pathway drives EGFR-TKI resistance that could be

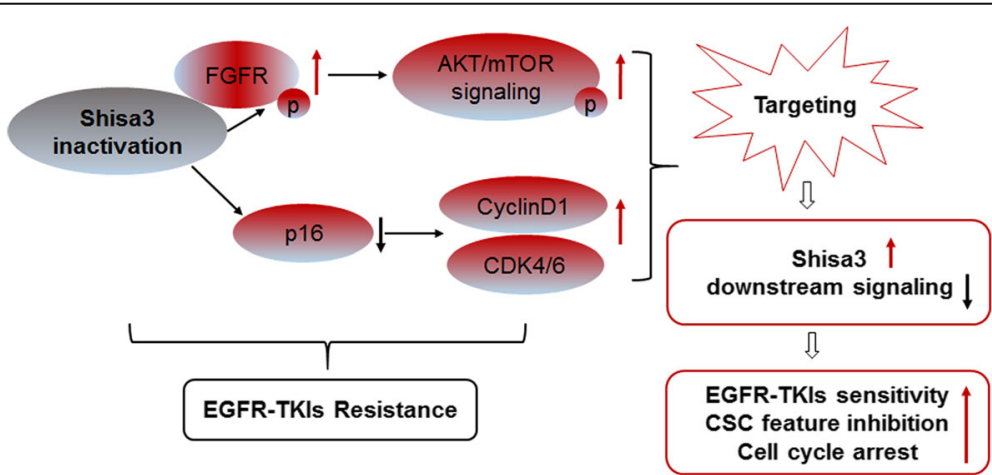

Fig. 7 A schematic of shisa3-dependent FGFR/AKT/mTOR and cell cycle signaling that mediates EGFR-TKIs response and CSC phenotype in lung adenocarcinoma 
triggered by a variety of signaling [14]. FGFR induced by $\mathrm{N}$-cadherin resulted in the phosphorylation of ERK and AKT to promote epithelial-mesenchymal transition (EMT) and CSC properties [29]. Our current findings suggest that shisa3 overcame EGFR-TKI resistance and CSC phenotypes by inactivating FGFR/AKT/mTOR and cell cycle signaling. We also found that EMT (down-regulation of E-cadherin, up-regulation of $\mathrm{N}$-cadherin and vimentin) existed in the PC9/ER cells with resistance to gefitinib and osimertinib, compared to the parental PC9 cells. In addition, Met and HER2 amplification have been reported to drive EGFR-TKIs resistance [21]. In consistent with previous study, we also detected higher expression of Met in the PC9/ER cells. In summary, several mechanisms like decreased of shisa3, increased of Met and EMT activation lead to EGFR-TKIs resistance.

mTOR inhibition has been reported to suppress tumor growth in lung cancer cells and patient-derived xenograft (PDX) models [30]. In addition, an mTOR inhibitor that mediated cell cycle arrest displayed antitumor activity in preclinical evidence [31]. The inhibition of cyclindependent kinase 4/6 in vivo demonstrated a better erlotinib response to suppress TKI drug resistance in esophageal squamous cell carcinoma [32]. Currently, we found that TKIs combined with inhibition of the cell cycle and $\mathrm{PI} 3 \mathrm{~K} / \mathrm{AKT} / \mathrm{mTOR}$ signaling dramatically suppressed tumor growth in PC9/ER xenografts related to shisa3 activation.

\section{Conclusions}

In summary, we demonstrated the crucial role of shisa3 in attenuating EGFR-TKI resistance, which is associated with CSC characteristics and the cell cycle. Our molecular studies indicated that shisa3 interacts with FGFR1/ FGFR3 to decrease the activation of these two receptors and their downstream AKT/mTOR pathway, resulting in restoration of EGFR-TKI sensitivity and suppression of CSC properties in lung adenocarcinoma. Based on these essential findings, we suggest that shisa3 or cotargeting FGFR/AKT/mTOR and cell cycle signaling may be an effective therapeutic strategy for overcoming resistance to gefitinib and osimertinib.

\section{Supplementary information}

Supplementary information accompanies this paper at https://doi.org/10. 1186/s13046-019-1486-3.

Additional file 1: Table S1. QRT-PCR primer sequences. Table S2. EGFR mutations in lung adenocarcinoma patients. Table S3. EGFR mutations in lung adenocarcinoma cells. Table S4. mTOR signaling pathway enrichment in the PC9 cells with shisa3 knock-down. Table S5. Cell cycle signaling pathway enrichment in the PC9 cells with shisa3 knock-down. Figure S1. PC9/ER cells resistant to EGFR-TKls induce CSC phenotype. Figure S2. Shisa3 decreases EGFR-TKI resistance and inhibits the CSC phenotype. Figure S3. EGFR-TKI inhibits tumor growth derived from PC9 cells in vivo.

\section{Abbreviations}

CDS: Coding sequence; CSCs: Cancer stem cells; DFS: Disease-free survival; EGFR: Epidermal growth factor receptor; EMT: Epithelial-mesenchymal transition; FGFR: Fibroblast growth factor receptor; IC50: Half maximal inhibitory concentration; IHC: Immunohistochemistry; MAPK: Mitogen activated protein kinase; MET: Mesenchymal epithelial transition receptor; NCBI: The national center for biotechnology information; Nod-scid: Nonobese diabetic-severe combined immunodeficiency; NSCLC: Non-small cell lung cancer; OS: Overall survival; PI3K: Phosphoinositide-3-kinase; PR: Partial response; RTK: Receptor tyrosine kinase; SD: Stable disease; STAT3: Signal transducer and activator of transcription 3; TKl: Tyrosine kinase inhibitor

\section{Acknowledgements}

Not applicable.

\section{Author's contributions}

The project was conceived by YY, NW and YYM. YYM and JHS designed the strategy, interpreted the data and wrote the manuscript. JHS performed the experiments. JWB constructed the vector. CL and SLL provided clinical samples and clinical information. YX contributed to technical support. YY and YYM provided advice and supervised the research. All authors read and approved the final manuscript.

\section{Funding}

This work was supported by National Key R\&D Program of China (No. 2018YFC0910700), National Natural Science Foundation of China (No.81772494 and 81972842), Beijing Natural Science Foundation (No. 7192036), Beijing Municipal Science \& Technology Commission (No. Z161100000516063), Special Fund of Beijing Municipal Administration of Hospitals Clinical Medicine Development (No. XMLX201841), Beijing Human Resources and Social Security Bureau (Beijing Millions of Talents Project, 2018A05), Peking University Medicine Seed Fund for Interdisciplinary Research (BMU2018MX008).

\section{Availability of data and materials}

All data generated or analyzed during this study are included in this article. and its additional files.

Ethics approval and consent to participate

The research was approved by the Ethics Committee of Peking University Cancer Hospital. All animal studies were performed in accordance with the animal experimental guidelines of Peking University Cancer Hospital.

\section{Consent for publication}

Not applicable.

\section{Competing interests}

The authors have declared that no competing interest exists.

Received: 30 August 2019 Accepted: 22 November 2019

Published online: 04 December 2019

\section{References}

1. Jackman D, Pao W, Riely GJ, Engelman JA, Kris MG, Janne PA, et al. Clinical definition of acquired resistance to epidermal growth factor receptor tyrosine kinase inhibitors in non-small-cell lung cancer. J Clin Oncol. 2010; 28:357-60.

2. Codd AS, Kanaseki T, Torigo T, Tabi Z. Cancer stem cells as targets for immunotherapy. Immunology. 2018;153:304-14.

3. MacDonagh L, Gray SG, Breen E, Cuffe S, Finn SP, O'Byrne KJ, et al. Lung cancer stem cells: the root of resistance. Cancer Lett. 2016;372:147-56.

4. Batlle E, Clevers H. Cancer stem cells revisited. Nat Med. 2017;23:1124-34.

5. Kharkar PS. Cancer stem cell (CSC) inhibitors: a review of recent patents (2012-2015). Expert Opin Ther Pat. 2017;27:753-61.

6. Chiu CF, Chang YW, Kuo KT, Shen YS, Liu CY, Yu YH, et al. NF-kappaB-driven suppression of FOXO3a contributes to EGFR mutation-independent gefitinib resistance. Proc Natl Acad Sci U S A. 2016;113:E2526-35.

7. Yamamoto A, Nagano T, Takehara S, Hibi M, Aizawa S. Shisa promotes head formation through the inhibition of receptor protein maturation for the caudalizing factors, Wnt and FGF. Cell. 2005;120:223-35. 
8. Wang K, Ji W, Yu Y, Li Z, Niu X, Xia W, et al. FGFR1-ERK1/2-SOX2 axis promotes cell proliferation, epithelial-mesenchymal transition, and metastasis in FGFR1-amplified lung cancer. Oncogene. 2018;37:5340-54.

9. Lu T, Li Z, Yang Y, Ji W, Yu Y, Niu X, et al. The hippo/YAP1 pathway interacts with FGFR1 signaling to maintain stemness in lung cancer. Cancer Lett. 2018:423:36-46.

10. Chen CC, Chen HY, Su KY, Hong QS, Yan BS, Chen CH, et al. Shisa3 is associated with prolonged survival through promoting beta-catenin degradation in lung cancer. Am J Respir Crit Care Med. 2014;190:433-44.

11. Pao W, Miller VA, Politi KA, Riely GJ, Somwar R, Zakowski MF, et al. Acquired resistance of lung adenocarcinomas to gefitinib or erlotinib is associated with a second mutation in the EGFR kinase domain. PLoS Med. 2005;2:e73.

12. Thress KS, Paweletz CP, Felip E, Cho BC, Stetson D, Dougherty B, et al. Acquired EGFR C797S mutation mediates resistance to AZD9291 in nonsmall cell lung cancer harboring EGFR T790M. Nat Med. 2015;21:560-2.

13. Liu Q, Yu S, Zhao W, Qin S, Chu Q, Wu K. EGFR-TKls resistance via EGFRindependent signaling pathways. Mol Cancer. 2018;17:53.

14. Jacobsen K, Bertran-Alamillo J, Molina MA, Teixido C, Karachaliou N, Pedersen $\mathrm{MH}$, et al. Convergent Akt activation drives acquired EGFR inhibitor resistance in lung cancer. Nat Commun. 2017:8:410.

15. Yu HA, Suzawa K, Jordan E, Zehir A, Ni A, Kim R, et al. Concurrent alterations in EGFR-mutant lung cancers associated with resistance to EGFR kinase inhibitors and characterization of MTOR as a mediator of resistance. Clin Cancer Res. 2018;24:3108-18.

16. Chang TH, Tsai MF, Su KY, Wu SG, Huang CP, Yu SL, et al. Slug confers resistance to the epidermal growth factor receptor tyrosine kinase inhibitor. Am J Respir Crit Care Med. 2011;183:1071-9.

17. Furugaki K, Fukumura J, Iwai T, Yorozu K, Kurasawa M, Yanagisawa M, et al Impact of bevacizumab in combination with erlotinib on EGFR-mutated non-small cell lung cancer xenograft models with T790M mutation or MET amplification. Int J Cancer. 2016;138:1024-32.

18. Piotrowska Z, Isozaki H, Lennerz JK, Gainor JF, Lennes IT, Zhu WW, et al. Landscape of acquired resistance to Osimertinib in EGFR-mutant NSCLC and clinical validation of combined EGFR and RET inhibition with Osimertinib and BLU-667 for acquired RET fusion. Cancer Discov. 2018;8:1529-39.

19. Shien K, Toyooka S, Yamamoto H, Soh J, Jida M, Thu KL, et al. Acquired resistance to EGFR inhibitors is associated with a manifestation of stem celllike properties in cancer cells. Cancer Res. 2013;73:3051-61.

20. Yu F, Liu JB, Wu ZJ, Xie WT, Zhong XJ, Hou LK, et al. Tumor suppressive microRNA-124a inhibits stemness and enhances gefitinib sensitivity of nonsmall cell lung cancer cells by targeting ubiquitin-specific protease 14 . Cancer Lett. 2018:427:74-84.

21. Wu SG, Shih JY. Management of acquired resistance to EGFR TKI-targeted therapy in advanced non-small cell lung cancer. Mol Cancer. 2018;17:38.

22. Datta J, Damodaran S, Parks H, Ocrainiciuc C, Miya J, Yu L, et al. Akt activation mediates acquired resistance to fibroblast growth factor receptor inhibitor BGJ398. Mol Cancer Ther. 2017;16:614-24.

23. Del Re M, Arrigoni E, Restante G, Passaro A, Rofi E, Crucitta S, et al. Concise review: resistance to tyrosine kinase inhibitors in non-small cell lung Cancer: the role of Cancer stem cells. Stem Cells. 2018;36:633-40.

24. Takahashi-Yanaga F, Kahn M. Targeting Wnt signaling: can we safely eradicate cancer stem cells? Clin Cancer Res. 2010;16:3153-62.

25. Takebe N, Harris PJ, Warren RQ, Ivy SP. Targeting cancer stem cells by inhibiting Wnt, notch, and hedgehog pathways. Nat Rev Clin Oncol. 2011;8: 97-106.

26. Maehara O, Suda G, Natsuizaka M, Ohnishi S, Komatsu Y, Sato F, et al. Fibroblast growth factor-2-mediated FGFR/Erk signaling supports maintenance of cancer stem-like cells in esophageal squamous cell carcinoma. Carcinogenesis. 2017:38:1073-83.

27. Zhou J, Wulfkuhle J, Zhang H, Gu P, Yang Y, Deng J, et al. Activation of the PTEN/mTOR/STAT3 pathway in breast cancer stem-like cells is required for viability and maintenance. Proc Natl Acad Sci U S A. 2007;104:16158-63.

28. Lai $Y, Y u X$, Lin $X$, He S. Inhibition of mTOR sensitizes breast cancer stem cells to radiation-induced repression of self-renewal through the regulation of MnSOD and Akt. Int J Mol Med. 2016;37:369-77.

29. Qian X, Anzovino A, Kim S, Suyama K, Yao J, Hulit J, et al. N-cadherin/FGFR promotes metastasis through epithelial-to-mesenchymal transition and stem/progenitor cell-like properties. Oncogene. 2014;33:3411-21.

30. Li R, Ding C, Zhang J, Xie M, Park D, Ding Y, et al. Modulation of Bax and mTOR for Cancer therapeutics. Cancer Res. 2017:77:3001-12.
31. Chen BW, Chen W, Liang H, Liu H, Liang C, Zhi X, et al. Inhibition of mTORC2 induces cell-cycle arrest and enhances the cytotoxicity of doxorubicin by suppressing MDR1 expression in HCC cells. Mol Cancer Ther. 2015:14:1805-15.

32. Zhou J, Wu Z, Wong G, Pectasides E, Nagaraja A, Stachler M, et al. CDK4/6 or MAPK blockade enhances efficacy of EGFR inhibition in oesophageal squamous cell carcinoma. Nat Commun. 2017;8:13897.

\section{Publisher's Note}

Springer Nature remains neutral with regard to jurisdictional claims in published maps and institutional affiliations.
Ready to submit your research? Choose BMC and benefit from:

- fast, convenient online submission

- thorough peer review by experienced researchers in your field

- rapid publication on acceptance

- support for research data, including large and complex data types

- gold Open Access which fosters wider collaboration and increased citations

- maximum visibility for your research: over $100 \mathrm{M}$ website views per year

At BMC, research is always in progress.

Learn more biomedcentral.com/submissions 